\title{
Last Measurable Excretion Rate Normalized by Body Mass Index
}

National Cancer Institute

\section{Source}

National Cancer Institute. Last Measurable Excretion Rate Normalized by Body Mass

Index. NCl Thesaurus. Code C92391.

The last measurable (positive) excretion rate divided by the body mass index. 